\title{
Schwerpunktthema: Tumorsyndrome
}

medgen $2017 \cdot 29: 276-282$

https://doi.org/10.1007/s11825-017-0154-2

Online publiziert: 8. November 2017

(c) Der/die Autor(en) 2017. Dieser Artikel ist eine Open-Access-Publikation.

CrossMark

\author{
Verena Steinke-Lange - Kerstin Becker · Anne Behnecke - Teresa Neuhann · \\ Elke Holinski-Feder
}

Medizinisch Genetisches Zentrum, München, Deutschland

\section{Syndrome mit breitem Tumorspektrum}

Mutation erfüllen, wurde eine ursächliche Methylierung des KLLN-Promotors gefunden [3], bei $10 \%$ lagen Veränderungen in $S D H B, S D H C$ und $S D H D$ vor, bei weiteren $10 \%$ Mutationen in PIK3CA oder $A K T 1$, selten in SEC23B (letztere v. a. bei Patienten mit Schilddrüsenkarzinom) [18, 31]. Die Inzidenz des CS wird auf mindestens 1:200.000 geschätzt, bei über der Hälfte handelt es sich um sporadische Fälle, der Anteil der Neumutationen ist unklar.

Weitere PTEN-assoziierte Krankheitsbilder werden zusammen mit dem CS unter dem Begriff des PTEN-Hamartoma-Tumor-Syndroms (PHTS) zusammengefasst: Zum Bannayan-RileyRuvalcaba-Syndrom (BRRS) gehören bereits im Kindesalter eine Makrozephalie, gastrointestinale hamartomatöse Polypen, Lipome, hyperpigmentierte Flecken der Glans penis sowie in ca. $50 \%$ eine Intelligenzminderung. Bei Patienten mit einem BRRS und einer PTENMutation wird von einem ähnlichen Tumorspektrum wie beim CS ausgegangen. Zum Teil wurden PTEN-Mutationen bei
Patienten mit einem Proteus-like-Syndrom beschrieben (s. Beitrag von Spier „Überwuchssyndrome durch Mutationsmosaike im PI3K-AKT-Signalweg" in diesem Schwerpunkt).

Es gibt inzwischen eine Reihe von Studien, die sich den Tumorrisiken beim CS widmen. Allerdings unterliegen viele dieser Studien einem Bias bei der Erfassung der Patienten, sodass die ermittelten Risiken nur eingeschränkt gültig sind.

Bei etwa 2/3 der CS-Patientinnen finden sich Fibroadenome der Brust. Das Lebenszeitrisiko (LZR) für Brustkrebs wird mit $85 \%$ angegeben, die Hälfte der Betroffenen erkrankt bereits vor dem 50. Lebensjahr (LJ) [25], eine Risikoerhöhung für männliche Anlageträger wird nicht beschrieben. Dazu kommen gutartige uterine Fibrome sowie ein erhöhtes LZR (ca. $28 \%$ ) für prä- und postmenopausale Endometriumkarzinome [25].

Eine Struma nodosa oder follikuläre Adenome der Schilddrüse finden sich bei etwa 3/4 der CS-Patienten, für Schilddrüsenkarzinome (meist follikuläre oder
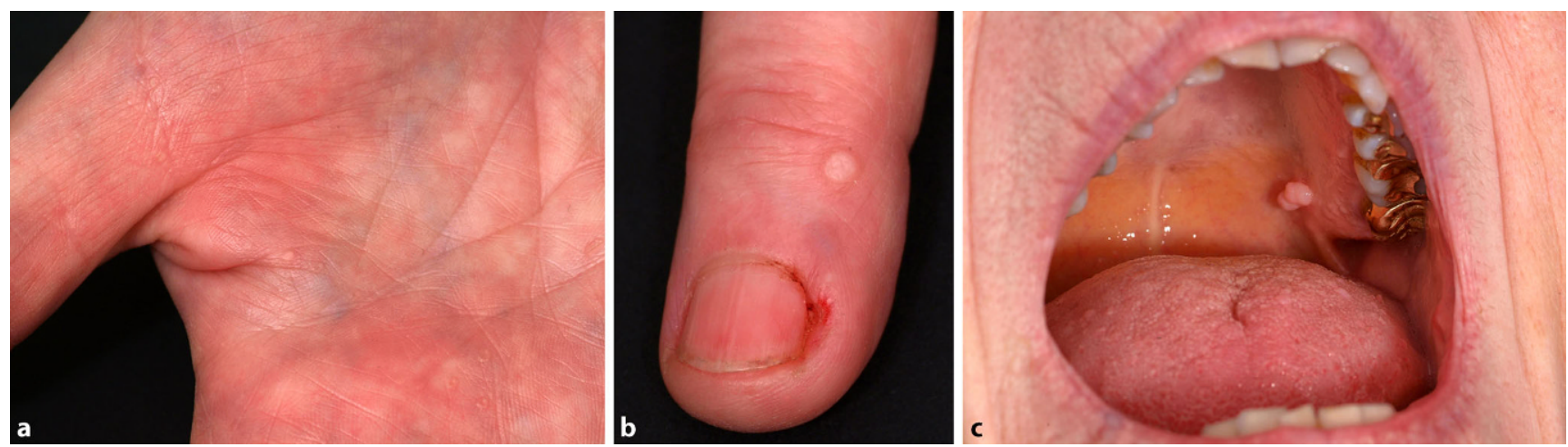

Abb. 1a-c $\Delta$ Mukokutane, papillomatöse Hautveränderungen bei einer 63-jährigen Patientin mit bilateralem Mammakarzinom (44 und 51 Jahre), gastrointestinaler Polyposis, Makrozephalie und Nachweis einer pathogenen PTEN-KeimbahnMutation (Quelle: Institut für Humangenetik, Universitätsklinikum Bonn) 


\section{Tab. 1 Früherkennungsempfehlungen für das PHTS (einschließlich CS) [9]}

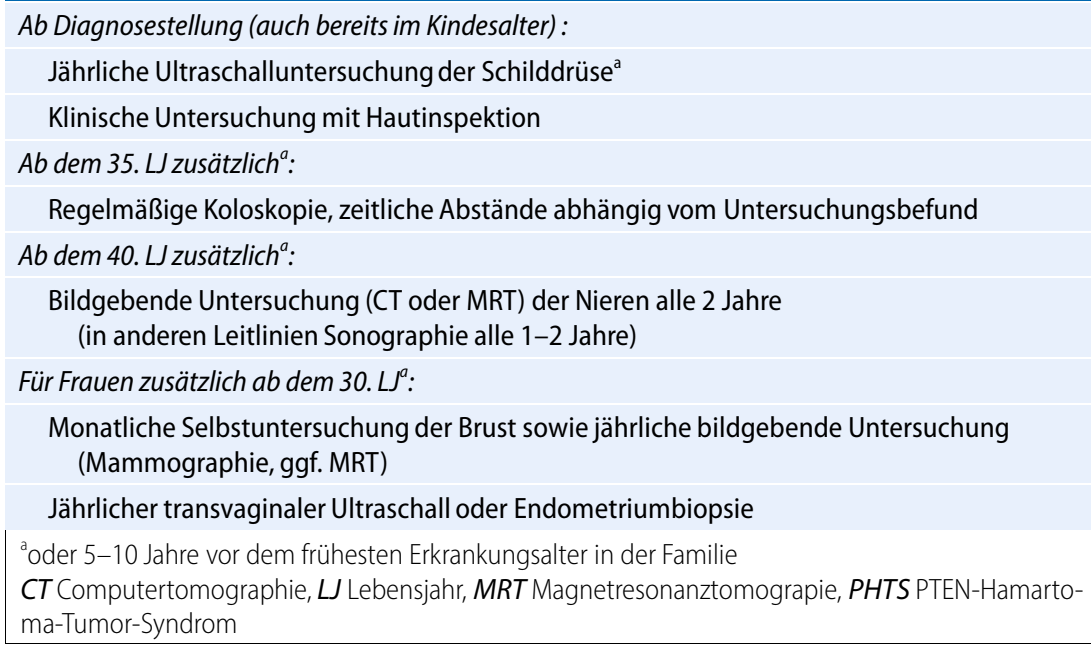

seltener papilläre, jedoch nie medulläre) besteht ein LZR von etwa $35 \%$ [25].

Gastrointestinale Polypen finden sich bei über $90 \%$, histologisch meist hamartomatöse Polypen ohne Entartungsrisiko. Es finden sich jedoch auch andere Polypenhistologien (insbesondere Ganglioneurome, juvenile Polypen, hyperplastische Polypen und Adenome), welche ein erhöhtes Darmkrebsrisiko (ca. 9\%) bedingen [25] und die klinische Abgrenzung $\mathrm{zu}$ anderen gastrointestinalen Polyposiserkrankungen erschweren.

Zum Tumorspektrum gehören auch (vorwiegend papilläre) Nierenzellkarzinome (LZR etwa $35 \%$, höher bei KLLNMethylierung) sowie Melanome (LZR > $5 \%)$. Tumoren des zentralen Nervensystems (ZNS) gehören nicht zum engeren Spektrum der CS-Tumoren. Allerdings wird das sehr seltene zerebelläre dysplastische Gangliozytom (LhermitteDuclos-Krankheit) im Erwachsenenalter als pathognomonisch für das CS angesehen. (Bei Kindern mit diesem Tumor liegen nur selten Keimbahnmutationen in PTEN vor.)

Ein beispielhafter Stammbaum einer Familie mit CS ist in $\mathbf{A} \mathbf{A b b} .2$ dargestellt.

Bei der klinischen Diagnosestellung und Abschätzung der Mutationsdetektionsrate kann ein Online-Punktesystem helfen, welches auf den Phänotypdaten sowie dem Erkrankungsalter einer prospektiven Studie an über 3000 Patienten mit klinischem CS beruht ([24]; www. lerner.ccf.org/gmi/ccscore). Ein in internationalen Leitlinien ab dem Alter von zehn Jahren vorgeschlagenes Früherkennungsprogramm ist in • Tab. 1 wiedergegeben.

Die Tumortherapie erfolgt entsprechend den Leitlinien, mTOR-Inhibitoren werden im Rahmen von Studien zur Behandlung von CS-Tumoren eingesetzt. Da eine erhöhte Neigung zur Keloidbildung besteht, sollten Hautveränderungen nur zurückhaltend (z. B. bei Malignomverdacht) entfernt werden. Viele Patienten mit CS zeigen zudem psychische Auffälligkeiten (z. B. Autismus).

\section{Peutz-Jeghers-Syndrom}

Die klassischen Symptome des autosomal-dominant vererbten Peutz-JeghersSyndroms (PJS) sind eine mukokutane Hyperpigmentierung und gastrointestinale Polyposis, die bereits im Kindesalter zu Anämie und Ileus-Symptomatik (Invagination) führen kann [12]. Die Inzidenz liegt bei 1:50.000-1:200.000 [2]. Peutz-Jeghers-Polypen (PJP) haben im Sinne hamartomatöser Polypen histologisch eine charakteristische baumartig verästelte Lamina muscularis mucosae. Sie können im gesamten Gastrointestinaltrakt vorliegen, insbesondere jedoch im Dünndarm und Dickdarm [26], selten auch extraintestinal (z. B. in den ableitenden Harnwegen, den Bronchien oder der Gallenblase).

Ursächlich sind Loss-of-functionVarianten in STK11, welches für eine Serin/Threonin-Kinase kodiert, die an
Tab. 2 Diagnosekriterien für das Peutz-

Jeghers-Syndrom (eines muss erfüllt sein)

- $\geq 2$ histologisch bestätigte PJP

- PJP $(\geq 1)$ bei einer Person mit positiver FA für ein PJS

- Charakteristische Hyperpigmentierung bei einer Person mit positiver FA für ein PJS

- PJP ( $\geq 1)$ bei einer Person mit charakteristischer Hyperpigmentierung

FA Familienanamnese, PJP Peutz-JeghersPolypen, PJS Peutz-Jeghers-Syndrom

der Regulation von Zellzykluskontrolle und Apoptose beteiligt ist. Eine ursächliche Veränderung wird bei $94 \%$ der Patienten in STK11 nachgewiesen, $25 \%$ sind Neumutationen [23]. Hinsichtlich der Genotyp-Phänotyp-Korrelation gibt es Hinweise, dass trunkierende Mutationen mit einer stärker ausgeprägten Polyposis und früheren Manifestation einhergehen [20]. Liegen diese in der Kinase-Domäne XI zeigen die Polypen zudem ein erhöhtes Dysplasierisiko [30].

Das bereits im jungen Erwachsenenalter erhöhte und ab dem 50. LJ zusätzlich deutlich ansteigende Karzinomrisiko betrifft mit $40 \%$ das Kolon, mit $30 \%$ den Magen und mit $13 \%$ den Dünndarm. Die Beteiligung der PJP als Vorläufer für diese Neoplasien ist umstritten. Das LZR für Pankreaskarzinome liegt bei $36 \%$, für Mammakarzinome bei $54 \%$ (auch prämenopausal), für Endometrium- und Zervixkarzinome bei ca. $10 \%[2,23]$. Auch ist eine Häufung von multifokalen gutartigen Ovarialtumoren beschrieben (ca. $21 \%$ ), histologisch meist sogenannte Sex-Cord-Tumoren mit annulären Tubuli (SCTAT). Männer mit PJS haben ein erhöhtes Risiko für meist gutartige Gonadentumoren (ca. 9\%), v. a. großzellige kalzifizierende Sertoli-Zell-Tumoren (LCCSCT), die bereits präpubertär entstehen können.

Bei 95 \% der Patienten finden sich bereits im Kindesalter melanozytäre Makulae, v. a. im Gesicht (perioral an der Grenze des Lippenrots, um die Augen und Nasenlöcher sowie intraoral), ggf. auch perianal und an den Fingern. Bis auf die intraoralen Hyperpigmentierungen verblassen sie meist bis zum Erwachsenenalter [2]. Die klinischen Diagnosekriterien sind in $\bullet$ Tab. 2 wiedergegeben. 
Ein internationales Expertenpanel hat 2010 Vorsorgeempfehlungen publiziert $[2,23]$, die in - Tab. 3 zusammengefasst sind.

\section{Juvenile Polyposis}

Beim autosomal-dominant vererbten juvenilen Polyposis-Syndrom (JPS) liegen histologisch juvenile Polypen (JP) des Dickdarms, seltener des Magens und des Dünndarms vor. Aufgrund der labilen Struktur der Polypen kann es zu chronischen gastrointestinalen Blutungen mit konsekutiver Anämie kommen, die im Kindesalter ggf. in einer Hypoproteinämie mit Gedeihstörung resultiert. Die Mehrzahl der Patienten wird bis zum 20. LJ symptomatisch [15], die Inzidenz wird auf ca. 1:100.000 geschätzt [13].

Ursächlich sind $\mathrm{zu}$ gleichen Teilen Sequenzvarianten in BMPR1A und SMAD4, ca. $50 \%$ sind Neumutationen [1]. BMPR1A ist ein Rezeptor an Zelloberflächen, zu den Liganden zählen unter anderem BMP und TGF- $\beta$. SMAD4 ist intrazellulär an der TGF- $\beta$ assoziierten Signalvermittlung beteiligt.

Die intrafamiliäre Variabilität des JPS ist hoch. Das LZR für Kolonkarzinome wird mit ca. $40 \%$ und ca. $20 \%$ für Magenkarzinome (insbesondere dann, wenn bereits Polypen des Magens vorhanden sind) angegeben, Pankreas- und Dünndarmkarzinome scheinen sehr selten vorzukommen.

Pathogene Varianten in SMAD4 sind mit einem höheren Risiko für Polypen des oberen Gastrointestinaltraktes (und damit einem höheren Risiko für Magenkarzinome) sowie ggf. einer hereditären hämorrhagischen Teleangiektasie (HHT/M. Osler) assoziiert, v. a. bei pathogenen Varianten in Exon 8-11 [10]. Hierbei liegen krankhafte Gefäßerweiterungen vor, die sich oft bereits im Kindesalter klinisch durch mukokutane Teleangiektasien, Nasenbluten und arteriovenöse Malformationen (AVM) in inneren Organen (v. a. Leber, Lunge, Gehirn, Darm) mit den entsprechenden Komplikationen zeigen [10]. Eine spezifische Veränderung in SMAD4 wurde auch als ursächlich für das MyhreSyndrom (Kleinwuchs und Aortendilatation) beschrieben [11]. Patienten mit

medgen 2017· 29:276-282 https://doi.org/10.1007/s11825-017-0154-2

(c) Der/die Autor(en) 2017. Dieser Artikel ist eine Open-Access-Publikation.

V. Steinke-Lange · K. Becker · A. Behnecke - T. Neuhann · E. Holinski-Feder Syndrome mit breitem Tumorspektrum

\section{Zusammenfassung}

Die klinische Diagnose erblicher Tumorsyndrome ist ein wesentlicher Bestandteil der humangenetischen Beratung. Bei seltenen Syndromen, die mit einem breiten Spektrum an Tumoren und phänotypischen Überschneidungen einhergehen, ist dies nicht immer einfach. In diesem Artikel sollen deshalb die typischen und wegweisenden Merkmale der wichtigsten seltenen Tumordispositionssyndrome mit breitem Tumorspektrum herausgearbeitet werden. Hierzu gehören der Carney-Komplex, das Cowden-Syndrom, die juvenile Polyposis, das Li-Fraumeni-Syndrom und das Peutz-Jeghers-Syndrom. Darüber hinaus sind auch die derzeit empfohlenen Früherkennungsuntersuchungen dargestellt, die meist eine interdisziplinäre Betreuung der Patienten erforderlich machen.

\section{Schlüsselwörter}

Carney-Komplex · Cowden-Syndrom · Juvenile Polyposis · Li-Fraumeni-Syndrom · PeutzJeghers-Syndrom

\section{Syndromes with a broad tumor spectrum}

\section{Abstract}

The clinical diagnosis of hereditary cancer syndromes is a significant component of genetic counseling. Rare cancer syndromes, which can be associated with a wide spectrum of tumor types and phenotypic overlap, may make this difficult. This article presents the leading characteristic and ground-breaking features of the most important rare cancer syndromes with a wide spectrum of tumor types, including Carney complex, Cowden syndrome, juvenile polyposis, Li-Fraumeni syndrome, and Peutz-Jeghers syndrome. Additionally, the current surveillance strategies are shown, which in most cases require an interdisciplinary approach to the patients.

\section{Keywords}

Carney complex - Cowden syndrome . Juvenile polyposis - Li-Fraumeni syndrome . Peutz-Jeghers syndrome einer Mikrodeletion 10q22-q23, die u. a. das BMPR1A- und PTEN-Gen enthält, können zusätzlich PTEN-assoziierte Symptome und einen sehr frühmanifesten Beginn der Symptomatik aufweisen.

Klinisch ist für die Diagnosestellung das Vorliegen mehrerer JP wegweisend (mehr als 5 JP im Kolorektum und/oder multiple JP im gesamten Gastrointestinaltrakt und/oder 1 JP bei positiver FA). Entscheidend für die Diagnose ist somit die korrekte histologische Beurteilung der Polypen.

Entsprechend angloamerikanischer Leitlinien wird etwa ab dem 15. LJ (bei Symptomen ggf. früher) eine Koloskopie und Ösophagogastroduodenoskopie empfohlen, die bei Polypennachweis jährlich, ansonsten alle 2-3 Jahre wiederholt werden sollte [17]. Bei massiver Polyposis sind ggf. chirurgische Maßnahmen nötig. Patienten mit einer SMAD4-Mutation benötigen zusätzliche
Vorsorgeuntersuchungen bezüglich der klinischen Manifestationen einer HHT und Aortopathie [29].

\section{Carney-Komplex}

Der Carney-Komplex (CNC) ist ein seltenes autosomal-dominant erbliches Tumorsyndrom, das sich durch das Vorliegen multipler endokriner und nicht endokriner Tumoren sowie Pigmentauffälligkeiten der Haut und Mukosa auszeichnet. Es ist nicht zu verwechseln mit der Carney-Trias (Paragangliome, gastrointestinale Stroma-Tumoren (GIST), pulmonale Chondrome). Die Diagnosestellung erfolgt meist im jungen Erwachsenenalter, selten früher oder nach dem 50. LJ [4, 22]. Circa 750 Patienten mit CNC sind weltweit erfasst, die Prävalenz ist unbekannt.

Ursächlich sind pathogene Varianten in PRKAR1A, das für die regulatorische 
Tab. 3 Früherkennungsuntersuchungen beim Peutz-Jeghers-Syndrom [2, 16, 23]

Ab dem 8. bzw. 18. L`:

Ösophagogastroduodenoskopie alle 3 Jahre

Kapselendoskopie alle 3 Jahre (Entfernung großer Polypen $>1,5 \mathrm{~cm}$ mittels Doppel-Ballon-Enteroskopie)

Koloskopie alle 3 Jahre

Ab dem 30. L zusätzlich:

Pankreasuntersuchung mittels MRCP oder Endosonographie alle 1-2 Jahre

Für Frauen ab dem 18. L zusätzlich:

Monatliche Selbstuntersuchung der Brust, halbjährliche klinische Untersuchung Jährlich transvaginaler Ultraschall und CA125-Bestimmung

Jährlich vaginale Untersuchung mit PAP-Abstrich

Für Frauen ab dem 25. L zusätzlich:

Jährliche bildgebende Untersuchung (MRT der Brust oder Mammographie)

Für Jungen/Männer ab Geburt zusätzlich:

Jährliche Hodenuntersuchung, ggf. Sonographie

aEine erste endoskopische Untersuchung wird mit 8 Jahren empfohlen. Bei Auffälligkeiten sollten anschließend 3-jährliche Kontrollen erfolgen, bei unauffälligem Befund erfolgt die nächste Untersuchung im Alter von 18 Jahren.

$\checkmark$ Lebensjahr, MRCP Magnetresonanz-Cholangiopankreatikografie, MRT Magnetresonanztomographie, PAP pankreatitisassoziiertes Protein

\section{Tab. 4 Diagnostische Kriterien für CNC}

\section{Hauptkriterien}

Gesprenkelte Pigmentauffälligkeiten der Haut

(mit typischer Lokalisation an Lippen, Augenwinkeln, Konjunktiven, vaginaler/ peniler Mukosa)

Myxome von Haut, Mukosa oder Herz

Myxomatose der Brust ${ }^{\mathrm{a}}$ (oder Hinweise darauf im MRT)

Primär pigmentierte noduläre adrenokortikale Krankheit (PPNAD) ${ }^{\mathrm{a}}$

oder entsprechende Hinweise im Dexamethason-Hemmtest

Akromegalie durch GH-sezernierendes Hypophysenadenom

Schilddrüsenkarzinom (in jedem Alter) oder multiple echoarme Schilddrüsenknoten (vor der Pubertät)

Großzellig kalzifizierender Sertoli-Zell-Tumor (LCCSCT) (oder charakteristische Kalzifikationen im Hodengewebe)

Psammomatöse melanotische Schwannome (PMS) ${ }^{a}$

Blauer Nävus, multiple epitheloide blaue Nävi ${ }^{a}$

Osteochondromyxome ${ }^{\mathrm{a}}$

Ergänzende Kriterien:

Eine erstgradig verwandte Person mit Carney-Komplex (CNC)

Nachweis einer inaktivierenden PRKAR1A-Mutation

${ }^{a}$ histologisch gesichert

Typ 1a-Untereinheit der Proteinkinase A (PKA) kodiert. Loss-of-function-Mutationen führen zu einem verstärkten $\mathrm{Si}$ gnal der PKA [22].

Bei mehr als $70 \%$ der CNC-Patienten ist eine Mutation in PRKAR1A nachweisbar. Krankheitsassoziiert sind auch PRKACA und PRKACB, in denen in Einzelfällen Kopienzahlveränderungen („copy number gains“) nachgewiesen wurden [8], sowie eine Region in 2p16.

Das Krankheitsbild ist sehr variabel mit einer nahezu 100\%igen Penetranz [8]. Hautauffälligkeiten wie Lentigines, epithelioide blaue Nävi, kutane Myxome oder seltener Café-au-lait-Flecken und Spitz-Nävi finden sich bei ca. $80 \%$ der Patienten. Die Lentigines (v. a. Gesicht, Lippen, Genitalbereich) liegen zum Teil schon bei Geburt vor bzw. treten in der Regel vor der Pubertät auf, nehmen an Zahl und Farbintensität in der Adoleszenz zu und können im höheren Erwachsenenalter wieder etwas verblassen. $\mathrm{Ku}$ tane Myxome (insbesondere an Augenlid, Gehörgang, Brustwarzen und Genitalbereich) finden sich bei ca. 30-55\%, die häufigste, nicht kutane Manifestation sind kardiale Myxome, die im Durchschnitt mit 20 Jahren und nur selten in der Kindheit auftreten. Sie verursachen mehr als $50 \%$ der Todesfälle, weshalb regelmäßige kardiologische Kontrolluntersuchungen empfohlen werden $[4,6]$.

Die in der Regel vor dem 30. LJ auftretende primär pigmentierte noduläre adrenokortikale Krankheit (PPNAD) (adrenokortikale Hyperplasie, die häufig ein ACTH-unabhängiges Cushing-Syndrom verursacht) ist der häufigste endokrine Tumor mit einer Prävalenz von 25-60 \% [22]. Selten sind Nebennierenrindenkarzinome beschrieben.

Bei bis zu $75 \%$ findet sich eine asymptomatische Erhöhung von GH, IGF-1 oder Prolaktin im Serum oder ein auffälliger OGTT (oraler Glukosetoleranztest), bei ca. $67 \%$ zeigt sich eine somatomammotrophe Hyperplasie und die Inzidenz einer Akromegalie aufgrund von GH-sezernierenden Hypophysenadenomen ( $\mathrm{ab}$ ca. 30. LJ) liegt bei ca. 10-12\% [4, 6]. Selten sind Prolaktinome.

Bis zu 60 \% der Patienten zeigen insbesondere in Kindheit/Adoleszenz eine Schilddrüsenbeteiligung in Form von unspezifischen zystischen Veränderungen (75\%), meist follikulären Adenomen ( $25 \%)$ und selten $(<10 \%)$ papillären oder follikulären Karzinomen [19, 21].

Bei $<10 \%$ finden sich im jungen Erwachsenenalter seltene Neoplasien der Nervenscheiden (psammomatöse melanotische Schwannome, PMS), meist im Bereich des Gastrointestinaltrakts, Grenzstrangs oder der Thoraxwand, die in ca. $10 \%$ maligne entarten [8].

Mehr als $75 \%$ der Männer mit einem CNC entwickeln großzellig kalzifizierende Sertoli-Zell-Tumoren (LCCSCT) [21].

Seltene Manifestationen sind duktale Adenome und myxoide Fibroadenome der Brust, Ovarialzysten und -tumoren (seröse Zystadenome, zystische Teratome), Osteochondromyxome (meist 


\section{Schwerpunktthema: Tumorsyndrome}

Tab. 5 Früherkennungsuntersuchungen beim CNC [8]

Jährliche Echokardiographie ab Kindesalter

Regelmäßige Hautuntersuchungen

GH-, Prolaktin-, IGF-1-Kontrollen ab Adoleszenz, ggf. weitere Untersuchungen auf Cushing-Syndrom

Screening für Schilddrüsentumoren

CT zur Detektion einer PPNAD, MRT der Hypophyse

MRT von Hirn/Wirbelsäule/Thorax/Abdomen/Retroperitoneum/Becken für PMS-Detektion

Bei Männern jährliche sonographische Untersuchung der Testes ab Kindesalter

Bei Frauen mind. einmal transabdominale sonographische Untersuchung der Ovarien

Bei präpubertären Kindern Kontrolle von Längenwachstum und Pubertätsentwicklung

CNC Carney-Komplex, CT Computertomographie, LU Lebensjahr, MRT Magnetresonanztomogra-

phie, PMS psammomatöse melanotische Schwannome, PPNAD primäre pigmentierte noduläre adrenokortikale Dysplasie

\section{Tab. 6 Revidierte Chompret-Kriterien (ein Kriterium muss erfüllt sein) [7]}

\section{Patient mit ...}

...einem Tumor aus dem LFS-Spektrum (prämenopausales Mammakarzinom, Weichteilsarkom, Osteosarkom, Hirntumor, Nebennierenrindenkarzinom, Leukämie, bronchoalveoläres Lungenkarzinom) vor dem 46. LJ und mindestens einem erst- oder zweitgradig Verwandten mit einem LFS-Tumor vor dem 56. LJ oder mit multiplen Tumorerkrankungen (ausgenommen Mammakarzinom, wenn der Indexpatient ebenfalls ein Mammakarzinom hat)

...multiplen Tumorerkrankungen, von denen zwei zum LFS-Tumorspektrum gehören und der erste vor dem 46. LJ auftrat (ausgenommen meta-/synchrone Mammakarzinome)

...Nebennierenrindenkarzinom, unabhängig von der FA

...Plexus-choroideus-Karzinom, unabhängig von der FA

...Rhabdomyosarkom vom embryonalen anaplastischen Subtyp, unabhängig von der FA

...Mammakarzinom vor dem 31. LJ, unabhängig von der FA

FA Familienanamnese, LFS Li-Fraumeni-Syndrom, $L$ Lebensjahr

\section{Tab. 7 Früherkennungsuntersuchungen beim LFS nach Villani et al. [28]}

\section{Kindesalter}

Ultraschalluntersuchung von Bauch und Becken alle 3-4 Monate

Laboruntersuchungen (Blutbild, BSG, LDH, 17-OH-Progesteron, Testosteron gesamt, Dehydroepiandrosteronsulfat, Androstendion, falls möglich Cortisolbestimmung im 24 h-Sammelurin) alle 3-4 Monate

Jährliches Ganzkörper-MRT und Schädel-MRT

Körperliche Untersuchung mit Wachstumsparametern,

Pubertätsentwicklung sowie neurologische Untersuchung alle 3-4 Monate

Ab dem Erwachsenenalter zusätzlich:

Monatlich Selbstuntersuchung der Brust, klinische Tastuntersuchung halbjährlich (ab dem Alter von 20-25 Jahre oder 5-10 Jahre vor der frühesten Brustkrebserkrankung in der Familie)

Jährliche MRT-Untersuchungen der Brust und jährliche Mammographie ${ }^{a}$

(20.-75. LJ oder 5-10 Jahre vor der frühesten Brustkrebserkrankung in der Familie),

Erwägung einer prophylaktischen Mastektomie

Ultraschalluntersuchung von Bauch und Becken alle 3-4 Monate

Koloskopie alle 2 Jahre

(ab dem 25. LJ oder 10 Jahre vor dem frühesten Erkrankungsalter in der Familie)

Jährliche dermatologische Untersuchung

${ }^{a}$ Aufgrund der Strahlenbelastung sollte die Mammographie sehr zurückhaltend eingesetzt und nach Möglichkeit eine MRT-Untersuchung durchgeführt werden

BSG Blutsenkungsgeschwindigkeit, $L D H$ Laktatdehydrogenase, $L$ Lebensjahr, MRT Magnetresonanztomographie
$<2$. LJ), hepatozelluläre Adenome, verschiedene Neoplasien des Pankreas und in Einzelfällen Kolon-, Magenkarzinome sowie peritoneale fibröse Histiozytome [8].

Der CNC stellt somit eine Differentialdiagnose dar zu: McCune-AlbrightSyndrom, PJS, PHTS, zur Neurofibromatose sowie $\mathrm{zu}$ anderen Phakomatosen und Hamartomatosen.

Die Diagnose CNC wird klinisch gestellt, wenn zwei der in $\bullet$ Tab. 4 aufgeführten Hauptkriterien oder ein Hauptkriterium und ein Nebenkriterium erfüllt sind [8].

Bislang gibt es keine Leitlinien für die Früherkennungsuntersuchungen bei $\mathrm{CNC}$, von einer Expertengruppe wurde das in $\bullet$ Tab. 5 dargestellte Vorgehen vorgeschlagen.

\section{Li-Fraumeni-Syndrom}

Im Kindesalter treten beim autosomal-dominant vererbten Li-FraumeniSyndrom (LFS) vor allem Sarkome, Nebennierenrindenkarzinome, Leukämien und ZNS-Tumoren auf, wohingegen im Erwachsenenalter bei Frauen das Risiko für Mammakarzinome stark im Vordergrund steht, gefolgt von Weichteilsarkomen und Osteosarkomen [14]. Die geschätzte Prävalenz liegt bei 1:5000-1:20.000.

TP53-Keimbahnmutationen sind hauptursächlich, wobei 7-20\% der Patienten Neumutationen aufweisen. Darüber hinaus sind in einzelnen Familien mit LFS auch Mutationen im CHEK2Gen beschrieben, der ursächliche $\mathrm{Zu}$ sammenhang ist jedoch nicht gesichert.

TP53 reguliert zahlreiche Gene, welche in Zellzykluskontrolle, Apoptose, DNA-Reparatur und Seneszenz involviert sind. Die pathogenen Sequenzvarianten umfassen Missensemutationen (ca. $73 \%$, meist in der DNA-Bindungsdomäne lokalisiert), Nonsensemutationen (ca. 9\%), Spleißmutationen (ca. 8\%), Frameshiftmutationen (6\%), Deletionen, Duplikationen und andere chromosomale Rearrangements [27]. Je nach Mutationstyp- und -lokalisation ergeben sich unterschiedliche Pathomechanismen wie „loss-of-function“, dominantnegativer Effekt, "gain-of-function“ [5]. 


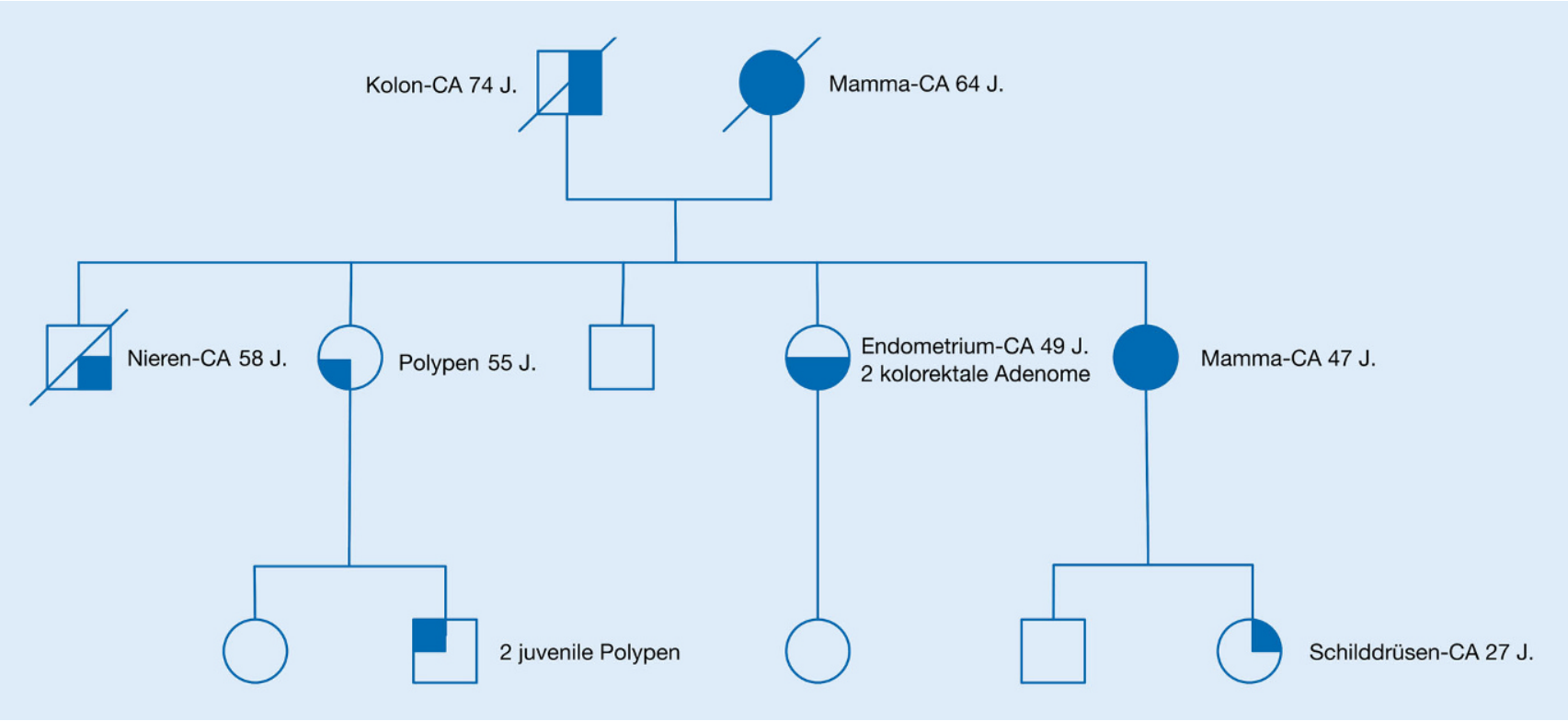

Abb. $2 \Delta$ Stammbaum einer Familie mit Cowden-Syndrom. In der Familie wären auch die Verdachtskriterien für erblichen Brust- und Eierstockkrebs und für den erblichen Darmkrebs ohne Polyposis (HNPCC/Lynch-Syndrom) erfüllt. Außerdem gibt es Hinweise auf eine juvenile Polyposis

Zur frühmanifesten Form des LFS gehören Sarkome, Nebennierenrindenkarzinome und hämatologische Tumoren (insbesondere „low hypodiploid“ ALL im Kindesalter, AML, MDS, seltener Lymphome) sowie ZNS-Tumore (Astrozytome, Glioblastome, Medulloblastome, Plexus-choroideus-Karzinome). Zur spätmanifesten Form des LFS gehören Weichteilsarkome, Osteosarkome, prämenopausale Mammakarzinome, ZNSTumore, Melanome, Lungenkarzinome, gastrointestinale Tumore, Schilddrüsenkarzinome und urogenitale Karzinome.

Für dominant-negative Missensemutationen liegt das Ersterkrankungsalter bei ca. 21 Jahren, bei „loss-of-function“ Mutationen hingegen bei 28,5 Jahren und bei genomischen Rearrangements bei 35,8 Jahren. Bei betroffenen Kindern mit Osteosarkomen, Hirntumoren oder Rhabdomyosarkomen finden sich hauptsächlich dominant-negative Missensemutationen, wohingegen bei Kindern mit Nebennierenrindenkarzinomen nicht dominant-negative Mutationen überwiegen, insbesondere p.(R158H). Die kumulative Tumorinzidenz bei 31-jährigen Frauen liegt bei 50 \% (vorwiegend Mammakarzinome), bei Männern wird dies mit 46 Jahren erreicht. Das Lebens- zeitrisiko liegt für Frauen bei nahezu $100 \%$, für Männer bei 73 \% [7]. Kumulative Inzidenzen bis zum 70 . LJ liegen für Weichteilsarkome bei $15 \% / 22 \%$ (Frauen/Männer), für Hirntumoren bei $6 \% / 19 \%$ und für Osteosarkome bei $5 \% / 11 \%$. Die kumulative Inzidenz von Mammakarzinomen bis zum 60. LJ beträgt $85 \%, 31 \%$ entwickeln ein kontralaterales Mammakarzinom, dies entspricht einer mit der von BRCA1/2Mutationsträgerinnen vergleichbaren Hochrisikosituation. Bei ca. $43 \%$ der LFS-Patienten traten multiple Primärtumoren auf, wobei ca. 30 \% der Patienten, die für den Ersttumor eine Strahlentherapie erhielten, Sekundärtumore im Strahlenfeld entwickelten.

Die diagnostischen Kriterien für das LFS sind in - Tab. 6 dargestellt. In der Klinik sollte man v.a. bei Patienten mit Nebennierenrindenkarzinom (Mutationsdetektionsrate [MDR] in TP53 bei Kindern $45 \%$ ), Plexus-choroideusKarzinom (MDR bei Kindern $42 \%$ ), Sarkomen (insbesondere Rhabdomyosarkomen vom embryonalen anaplastischen Subtyp [MDR ca. $52 \%$ ]), Mammakarzinom vor dem 31. LJ nach Ausschluss einer BRCA1/2-Mutation (MDR ca. 6-8\%), kolorektalem Karzinom in sehr jungem Alter und bei Patienten mit multiplen synchronen oder metachronen Tumorerkrankungen an das LFS denken.

In der Therapie führen eine Strahlentherapie oder bestimmte Chemotherapeutika, v. a. genotoxische Substanzen wie z.B. alkylierende Substanzen, zu einem deutlich erhöhten Risiko für Zweiterkrankungen (Sekundärtumoren im Bestrahlungsfeld, therapieassoziierte Leukämie/MDS). Dies muss sowohl bei der Wahl des Therapieregimes als auch der Früherkennungsmaßnahmen berücksichtigt werden.

Auch für das LFS gibt es keine Leitlinienempfehlung für die Früherkennung. In einer Studie wurde ein bislang nicht standardisiertes Früherkennungsprogramm empfohlen (• Tab. 7). Patienten, die an dem Screeningprogramm teilnahmen, hatten in der Studie eine verbesserte 5-Jahres-Überlebensrate von $88,8 \%$ versus $59,6 \%$ bei Nichtteilnehmern. Zudem sollten die Patienten auf allgemeine Anzeichen einer Tumorerkrankung (unerklärter Gewichtsverlust, Nachtschweiß, Abgeschlagenheit) und eine anfangs schmerzlose Schwellung als häufiges Symptom eines Sarkoms achten und bei Auffälligkeiten einen Arzt aufsuchen. 


\section{Fazit für die Praxis}

Seltene Tumorsyndrome mit breitem Tumorspektrum zeigen phänotypische Überlappungen mit zahlreichen anderen Syndromen, was die Diagnosestellung häufig erschwert. Für den klinischen Genetiker ist es deshalb hilfreich, sich die Hauptcharakteristika dieser Syndrome zu vergegenwärtigen, um klinische Hinweise auf diese Syndrome im Beratungskontext zu erkennen. Heutzutage kann die Diagnosestellung auch durch den Einsatz einer Multi-Gen-Analyse basierend auf einem Next Generation Sequencing (NGS)-Ansatz erleichtert werden.

\section{Korrespondenzadresse}

Dr. med. V. Steinke-Lange

Medizinisch Genetisches Zentrum

Bayerstraße 3-5, 80335 München, Deutschland Verena.Steinke-Lange@mgz-muenchen.de

\section{Einhaltung ethischer Richtlinien}

Interessenkonflikt. V. Steinke-Lange, K. Becker, A. Behnecke, T. Neuhann und E. Holinski-Feder geben an, dass kein Interessenkonflikt besteht.

Dieser Beitrag beinhaltet keine von den Autoren durchgeführten Studien an Menschen oder Tieren.

Open Access. Dieser Artikel wird unter der Creative Commons Namensnennung 4.0 International Lizenz (http://creativecommons.org/licenses/by/4.0/deed. de) veröffentlicht, welche die Nutzung, Vervielfältigung, Bearbeitung, Verbreitung und Wiedergabe in jeglichem Medium und Format erlaubt, sofern Sie den/die ursprünglichen Autor(en) und die Quelle ordnungsgemäßnennen, einen Linkzur Creative Commons Lizenz beifügen und angeben, ob Änderungen vorgenommen wurden

\section{Literatur}

1. Aretz S, Stienen D, Uhlhaas S et al (2007) High proportion of large genomic deletions and a genotype phenotype update in 80 unrelated families with juvenile polyposis syndrome. J Med Genet 44:702-709. https://doi.org/10.1136/jmg. 2007.052506

2. Beggs AD, Latchford AR, Vasen HFA et al (2010) Peutz-Jeghers syndrome: a systematic review and recommendations for management. Gut 59:975-986. https://doi.org/10.1136/gut.2009. 198499

3. Bennett KL, Mester J, Eng C (2010) Germline epigenetic regulation of KILLIN in Cowden and Cowden-like syndrome. JAMA 304:2724-2731. https://doi.org/10.1001/jama.2010.1877
4. Bertherat J, Horvath A, Groussin L et al (2009) Mutations in regulatory subunit type $1 \mathrm{~A}$ of cyclic adenosine 5'-monophosphate-dependent protein kinase (PRKAR1A): phenotype analysis in 353 patients and 80 different genotypes. J Clin Endocrinol Metab 94:2085-2091. https://doi.org/ 10.1210/jc.2008-2333

5. Bisio A, Ciribilli Y, Fronza Getal (2014) TP53 mutants in the tower of babel of cancer progression. Hum Mutat 35:689-701. https://doi.org/10.1002/ humu. 22514

6. Boikos SA, Stratakis CA (2007) Carney complex: the first 20 years. Curr Opin Oncol 19:24-29. https:// doi.org/10.1097/CCO.0b013e32801195eb

7. Bougeard G, Renaux-Petel M, Flaman J-M et al (2015) Revisiting Li-Fraumeni syndrome from TP53 mutation carriers. J Clin Oncol 33:2345-2352. https://doi.org/10.1200/JCO.2014.59.5728

8. Correa R, Salpea P, Stratakis CA (2015) Carney complex: an update. Eur J Endocrinol 173:M85-97. https://doi.org/10.1530/EJE-15-0209

9. Eng C (2001) PTEN Hamartoma Tumor Syndrome. In: Pagon RA, Adam MP, Ardinger $\mathrm{HH}$ et al (Hrsg) GeneReviews ${ }^{\circledR}$ [Internet]. University of Washington, Seattle (Updated 02.06.2016. Available from https://www.ncbi.nlm.nih.gov/books/NBK1488/)

10. Gallione CJ, Richards JA, Letteboer TGW et al (2006) SMAD4 mutations found in unselected HHT patients. J Med Genet 43:793-797. https://doi.org/ 10.1136/jmg.2006.041517

11. Le Goff C, Mahaut C, Abhyankar A et al (2011) Mutations at a single codon in mad homology 2 domain of SMAD4 cause Myhre syndrome. Nat Genet 44:85-88. https://doi.org/10.1038/ng.1016

12. Hinds R, Philp C, Hyer W, Fell JM (2004) Complications of childhood Peutz-Jeghers syndrome: implications for pediatric screening. J Pediatr Gastroenterol Nutr 39:219-220

13. Latchford AR, Neale K, Phillips RKS, Clark SK (2012) Juvenile polyposis syndrome: a study of genotype phenotype, and long-term outcome. Dis Colon Rectum 55:1038-1043. https://doi.org/10.1097/ DCR.0b013e31826278b3

14. Mai PL, Best AF, Peters JA et al (2016) Risks of first and subsequent cancers among TP53 mutation carriers in the National Cancer Institute Li-Fraumeni syndrome cohort. Cancer 122:3673-3681. https://doi.org/10.1002/cncr.30248

15. Manfredi M (2010) Hereditary Hamartomatous Polyposis Syndromes. Gastroenterol Hepatol 6:185-196

16. McGarrity TJ, Amos Cl, Baker MJ (2001) PeutzJeghers Syndrome. In: Pagon RA, Adam MP, Ardinger $\mathrm{HH}$ et al (Hrsg) GeneReviews ${ }^{\circledR}$ [Internet] University of Washington, Seattle (Updated 14.07.2016. Available from: https://www.ncbi nlm.nih.gov/books/NBK1266/)

17. National Comprehensive Cancer Network (2017) Genetic/Familial High-Risk Assessment: Colorectal (Version 1.2017). https://www.nccn.org/ professionals/physician gls/pdf/genetics colon. pdf. Zugegriffen: 14. Juli 2017

18. Orloff MS, He X, Peterson C et al (2013) Germline PIK3CA and AKT1 mutations in Cowden and Cowden-like syndromes. Am J Hum Genet 92:76-80. https://doi.org/10.1016/j.ajhg.2012.10. 021

19. Peiling Yang S, Ngeow J (2016) Familial nonmedullary thyroid cancer: unraveling the genetic maze. Endocr Relat Cancer 23:R577-R595. https:// doi.org/10.1530/ERC-16-0067

20. Salloch $H$, Reinacher-Schick $A$, Schulmann $K$ et al (2010) Truncating mutations in Peutz-Jeghers syndrome are associated with more polyps, surgical interventions and cancers. Int J Colorecta Dis 25:97-107. https://doi.org/10.1007/s00384009-0793-0

21. Stratakis CA, Kirschner LS, Carney JA (2001) Clinical and molecular features of the Carney complex: diagnostic criteria and recommendations for patient evaluation. J Clin Endocrinol Metab 86:4041-4046. https://doi.org/10.1210/jcem.86. 9.7903

22. Stratakis CA, Salpea P, Raygada M (2003) Carney Complex. In: Pagon RA, Adam MP, Ardinger HH et al (Hrsg) GeneReviews ${ }^{\circledR}$ [Internet]. University of Washington, Seattle (Updated 29.01.2015. Available from: https://www.ncbi.nlm.nih.gov/ books/NBK1286/)

23. Syngal S, Brand RE, Church JM et al (2015) ACG clinical guideline: genetic testing and management of hereditary gastrointestinal cancer syndromes. Am J Gastroenterol 110:223-262. https://doi.org/10.1038/ajg.2014.435

24. Tan M-H, Mester J, Peterson C et al (2011) A clinica scoring system for selection of patients for PTEN mutation testing is proposed on the basis of a prospective study of 3042 probands. Am J Hum Genet 88:42-56. https://doi.org/10.1016/j.ajhg. 2010.11 .013

25. Tan M-H, Mester JL, Ngeow J et al (2012) Lifetime cancer risks in individuals with germline PTEN mutations. Clin Cancer Res 18:400-407. https:// doi.org/10.1158/1078-0432.CCR-11-2283

26. Utsunomiya J, Gocho H, Miyanaga T et al (1975) Peutz-Jeghers syndrome: its natural course and management. Johns Hopkins Med J 136:71-82

27. Valdez JM, Nichols KE, Kesserwan C (2017) Li-Fraumeni syndrome: a paradigm for the understanding of hereditary cancer predisposition. Br J Haematol 176:539-552.https://doi.org/10.1111/bjh.14461

28. Villani A, Shore A, Wasserman JD et al (2016) Biochemical and imaging surveillance in germline TP53 mutation carriers with Li-Fraumeni syndrome: 11 year follow-up of a prospective observational study. Lancet Oncol 17:1295-1305. https://doi.org/10.1016/S1470-2045(16)30249-2

29. Wain KE, Ellingson MS, McDonald J et al (2014) Appreciating the broad clinical features of SMAD4 mutation carriers: a multicenter chart review. Genet Med 16:588-593. https://doi.org/10.1038/ gim.2014.5

30. Wang Z, Wu B, Mosig RA et al (2014) STK11 domain XI mutations: candidate genetic drivers leading to the development of dysplastic polyps in Peutz-Jeghers syndrome. Hum Mutat 35:851-858 https://doi.org/10.1002/humu.22549

31. Yehia L, Niazi F, Ni Y et al (2015) Germline heterozygous variants in SEC23B are associated with Cowden syndrome and enriched in apparently sporadic thyroid cancer. Am J Hum Genet 97:661-676. https://doi.org/10.1016/j.ajhg.2015. 10.001 